\title{
Detecting Nuclear Materials in Urban Environments Using Mobile Sensor Networks
}

\author{
R. Flanagan ${ }^{1}$, LJ Brandt ${ }^{2}$, MR Deinert ${ }^{1 *}$, and AG Osborne ${ }^{1 *}$ \\ ${ }^{1}$ Nuclear Science and Engineering, Colorado School of Mines \\ 1500 Illinois St. Golden, CO 80401 \\ ${ }^{2}$ United States Air Force Academy \\ Colorado Springs 80840-5002 \\ *mdeinert@mines.edu, osbornea@mines.edu
}

\begin{abstract}
Radiation detectors installed at major ports of entry are a key component of the overall strategy to protect countries from nuclear terrorism. While the goal of deploying these systems is to intercept special nuclear material as it enters the country, no detector system is fool proof. Mobile, distributed sensors have been proposed to detect nuclear materials in transit should portal monitors fail to prevent their entry in the first place. In large metropolitan areas a mobile distributed sensor network could be deployed using vehicle platforms such as taxis, Ubers and Lyfts which are already connected to communications infrastructure. However, the potential geographic coverage that could be achieved using a network of sensors mounted on commercial passenger vehicles has not been established. Here we evaluate how a mobile sensor network could perform in New York City using a combination of radiation transport and Geographic Information Systems. The Geographic Information System QGIS is used in conjunction with OpenStreetMap data to isolate roads and construct a grid over the streets. Vehicle paths are built using pickup and drop off data from Uber, and data from the New York State Department of Transportation.
\end{abstract}

KEYWORDS: distributed sensor network

\section{INTRODUCTION}

The increased potential for nuclear terrorism is a major obstacle to the deployment of nuclear power at a scale required for a transition to a sustainable and clean energy future. A fission weapon or dirty bomb detonated in a dense population center such as New York City would produce significant casualties in addition to widespread destruction and disruption. Several radiation detection systems, however, act as a first line defense for the United States. These systems include radiation detectors at airports, shipyards, commercial ports of entry [1,2], and roadway border crossings [1-3]. However, if these systems fail, or if non-official points of entry are used, special nuclear material could be smuggled into the country. Once inside, highways and streets can be used to transport the material to nearly any destination in the United States. 
The ease with which passengers and cargo can be moved across the United States presents a unique problem to protecting a city from nuclear terrorism. One option is to maintain a stationary detection grid with placement of detectors along entry points to the city. However, smart placement of those systems would be nontrivial [4,5], and although cities like Manhattan have a limited number of access routes such as bridges and tunnels, not all large cities have geographical features that simplify optimal deployment of detector systems.

Another approach to securing a city from nuclear terrorism would be a mobile radiation detection network. The effectiveness of a mobile detector fleet for locating a stationary source has been investigated where detector vehicles followed fixed or random paths [6]. Advanced machine learning algorithms have been shown to improve source characterization with multiple sources using mobile detectors [7]. Other studies [8] have focused on detecting mobile sources carried by individuals using stationary detectors and suggested that it might be possible to use mobile detectors attached to police patrol cars as well. The use of distributed sensor networks to detect stationary sources at highly populated events such as large sporting events was investigated in [9]. Taxis, limousines and ride-share services could provide an ideal platform for detector systems as these vehicles already possess power and communications infrastructure and are ubiquitous in most US cities. However, these vehicles follow unpredictable routes designated by passengers, drivers and modern navigation systems which can route cars according to congestion in near real-time. Due to the lack of control over detector movement, this could limit the effectiveness of this type of network in locating radiological threats. In the present work, we evaluate the potential for using mobile sensors mounted on Uber vehicles to detect a moving radioactive source in Manhattan, NY. The routes taken by the mobile detectors are estimated using historic Uber trip data combined with a route-finding algorithm. Geographical Information Systems (GIS) data on NYC buildings are used with a simple Green's function to model radiation transport. The effectiveness of the mobile sensor network is shown as a function of source strength and speed as well as the number of detector vehicles.

\section{METHODS}

The approach presented in this work simulates a single mobile radioactive source and a number of mobile radiation detectors mounted on Uber vehicles moving through streets in Manhattan, NY. Manhattan buildings' geometries in a Geographical Information Systems (GIS) format shapefile were obtained from OpenStreetMap [10]. Passenger pickup and drop-off zones and time stamps for 12/03/2018 Uber traffic were downloaded from the New York Taxi and Limousine Commission [11] and were randomly sampled to generate exact pickup and drop-off locations. The routes taken by each detector vehicle were computed by combining the pickup and drop-off locations and time stamps with the route-finding algorithm provided by the pyroute 3 library [12]. The route of the source vehicle was also determined using pyroute 3 and manually specifying an origin near the South-East Manhattan coast and Madison Square Gardens as a destination. The source and detector routes were discretized into equally spaced time indexes with a $\Delta t$ of $10 \mathrm{~s}$. The detector routes moved at a constant vehicle speed, which was computed using the total route length and duration. The source route incorporated a $10 \%$ random stop chance for each segment of the trip. The duration of the stops ranged from 1-10 seconds to simulate traffic conditions. Geometric data are stored and manipulated using the Shapely [13] python library.

The simulated radioactive source is ${ }^{60} \mathrm{Co}$ with a strength of 0.1 and $0.5 \mathrm{Ci}$, emitting 1.17 and $1.33 \mathrm{MeV}$ gammas. ${ }^{60} \mathrm{Co}$ was chosen because it is a characteristic nuclear material for dirty bombs $[14,15,16]$. In the simulations the source is shielded by $3 \mathrm{~cm}$ of lead, and additional shielding by its delivery vehicle is assumed to be 0.66 meters of air and $3 \mathrm{~cm}$ of steel. The gamma ray flux from the source at a given distance is approximated by Eq. 1, which accounts for both the inverse square law and attenuation: 


$$
F(r)=\frac{S e^{-r \mu_{\text {air }}}}{4 \pi r^{2}}
$$

Where $F(r)$ (counts $/ \mathrm{m}^{2}$-min) is the strength of the source at distance $\mathrm{r}(\mathrm{m}), \mu_{\text {air }}\left(\mathrm{m}^{-1}\right)$ is the linear attenuation coefficient for gammas in air, and $\mathrm{S}$ (counts/min) is the strength of the source after being attenuated by the shielding. The maximum distance at which the source can be detected is assumed to be at least three standard deviations above the background gamma radiation count rate for Manhattan $(\sim 5000$ counts/s) for a detector with an area of $0.0225 \mathrm{~m} 2$ and efficiency of $1 \%$ for gamma rays. This value was extracted from the RadNet service [17].

Once the source and detector routes are constructed and broken into points with timestamps, these points are used to create a Sort Tile Recursive Tree (STRtree) using the Shapely python library. The STRtree allows for quick querying of information about the points in relation to each other. For each point in the source path, the STRtree is queried to determine which detectors are in range of the source at the given timestamp. For each detector in range, the distance from the source to the detector is calculated and the amount of counts per second the detector measures is recorded. Buildings intersecting the path between source and detector are assumed to absorb all radiation, reducing the signal to zero. In this work, radiation transport through traffic other than the source and detector vehicles was ignored.

\section{RESULTS}

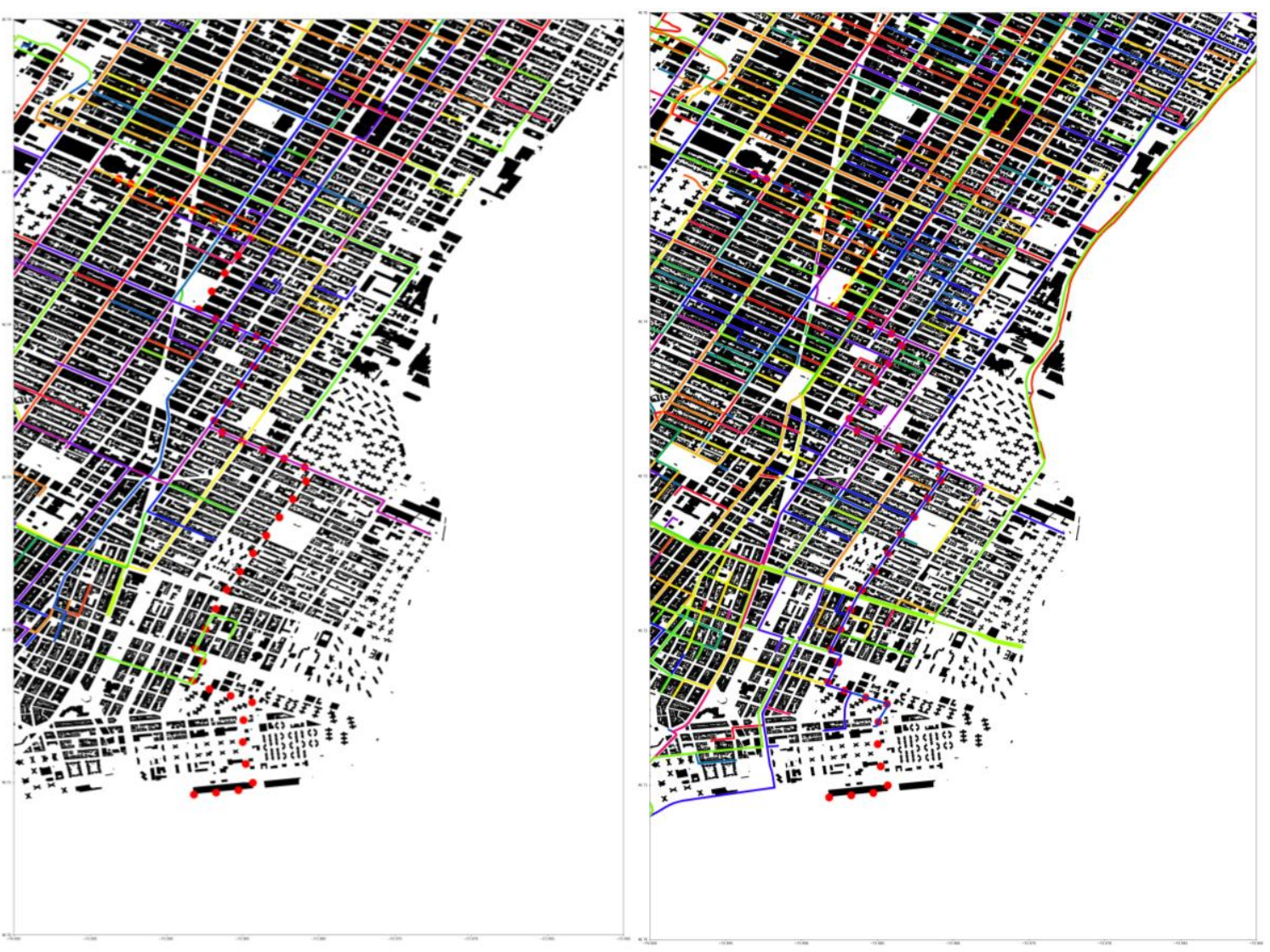

Figure 1. Visual of 100 and 500 Uber routes on Dec. $3^{\text {rd }}, 2018$. The solid lines represent the Uber traffic. The red dotted line represents the source path. All journeys begin and end inside the 20 -minute duration. Journeys that start or finish outside the duration were excluded. 
Generated routes during December 3rd, 2018 from 3:00pm until 3:20pm are shown in Fig. 1. While a total of 3500 Uber journeys were made in that duration, the number of routes sampled for performing the simulation was varied in order to evaluate the detector network performance with increasing numbers of detector vehicles. Figure 1 illustrates Uber tracks for 100 and 500 sampled routes. The solid colored lines represent the Uber tracks, and the red dotted line is the source path. Buildings are shown as solid black objects, and the white space represents roads, alleyways, parks, parking lots, and other space unoccupied by a building. Although Fig. 1 shows that increasing the number of Uber routes sampled increases the cumulative coverage of the city and that the source route intersects several detector (Uber) routes while moving from the shore to Madison Square Gardens, this does not guarantee that the source and a given detector are coincident.

Table I summarizes results from 12 simulated cases with source speeds that varied from 2 to $15 \mathrm{~m} / \mathrm{s}$, strengths of either 0.1 or $0.5 \mathrm{Ci}$, and with either 200 or 400 mobile detectors deployed. In each case, the number of times a source was detected during its transit and the integrated counts across all detectors and source route duration were recorded.

Table I. Case Study Parameters and Results: 12 cases were simulated with varying source speed and strength for 200 or 400 detectors. Source paths were identical in each case. Mobile sensors followed 200 or 400 identical routes.

\begin{tabular}{|c|c|c|c|c|c|c|}
\hline Case Number & $\begin{array}{c}\text { Speed of } \\
\text { Source (m/s) }\end{array}$ & $\begin{array}{c}\text { Speed of } \\
\text { Source(mph) }\end{array}$ & $\begin{array}{c}\text { Strength of } \\
\text { Source (Ci) }\end{array}$ & $\begin{array}{c}\text { Number of Mobile } \\
\text { Detectors }\end{array}$ & $\begin{array}{c}\text { Detection } \\
\text { Occurrences }\end{array}$ & $\begin{array}{c}\text { Total } \\
\text { Counts }\end{array}$ \\
\hline 1 & 2 & 4.47 & 0.1 & 200 & 0 & 0 \\
\hline 2 & 10 & 22.37 & 0.1 & 200 & 8 & $2.35 \times 10^{5}$ \\
\hline 3 & 15 & 33.55 & 0.1 & 200 & 0 & 0 \\
\hline 4 & 2 & 4.47 & 0.5 & 200 & 0 & 0 \\
\hline 5 & 10 & 22.37 & 0.5 & 200 & 16 & $1.74 \times 10^{6}$ \\
\hline 6 & 15 & 33.55 & 0.5 & 200 & 0 & 0 \\
\hline 7 & 2 & 4.47 & 0.1 & 400 & 9 & $8.37 \times 10^{5}$ \\
\hline 8 & 10 & 22.37 & 0.1 & 400 & 7 & $5.82 \times 10^{5}$ \\
\hline 9 & 15 & 33.55 & 0.1 & 400 & 0 & 0 \\
\hline 10 & 2 & 4.47 & 0.5 & 400 & 26 & $2.31 \times 10^{7}$ \\
\hline 11 & 10 & 22.37 & 0.5 & 400 & 17 & $8.13 \times 10^{6}$ \\
\hline 12 & 15 & 33.55 & 0.5 & 400 & 0 & 0 \\
\hline
\end{tabular}

Figure 2 shows the count rate that a fleet of 200 detectors following historic Uber routes for during December 3rd, 2018 from 3:00pm until 3:20pm would measure from a 0.1 and $0.5 \mathrm{Ci}$ source moving at $10 \mathrm{~m} / \mathrm{s}$ as a function of position. Count rates shown are summed over all detectors in range. Figure 3 shows the count rate for 400 detectors and a source speed of $2 \mathrm{~m} / \mathrm{s}$, and Fig. 4 shows the count rate for 400 detectors and $10 \mathrm{~m} / \mathrm{s}$ source speed. 

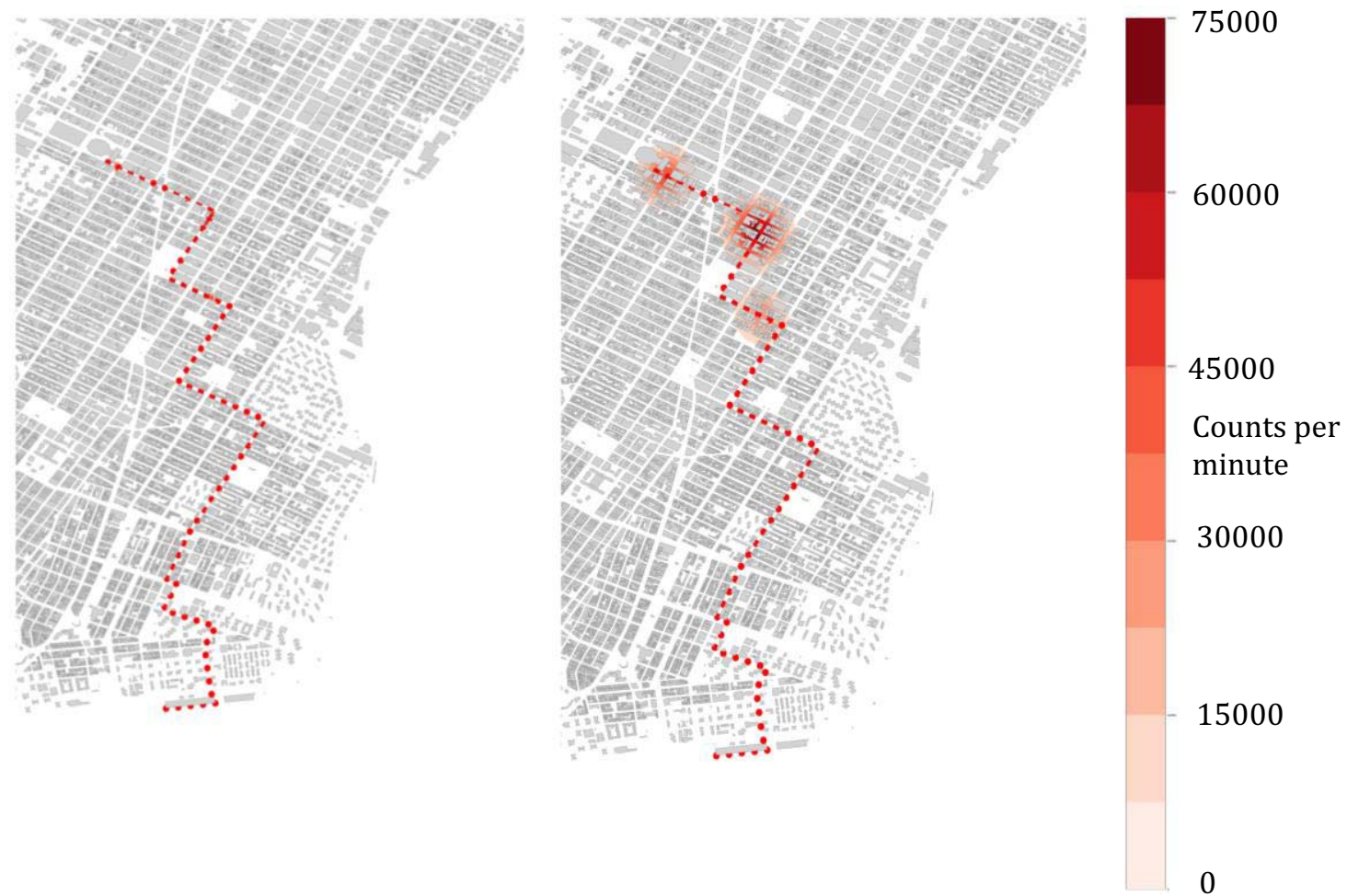

Figure 2. Detection heat maps. Maps show counts per minute detected from 200-vehicle mobile detector array of a $0.1 \mathrm{Ci} \mathrm{Co-60} \mathrm{(left)} \mathrm{and} \mathrm{a} 0.5 \mathrm{Ci} \mathrm{Co-60} \mathrm{(right)} \mathrm{source} \mathrm{moving} \mathrm{at} 10 \mathrm{~m} / \mathrm{s}$.
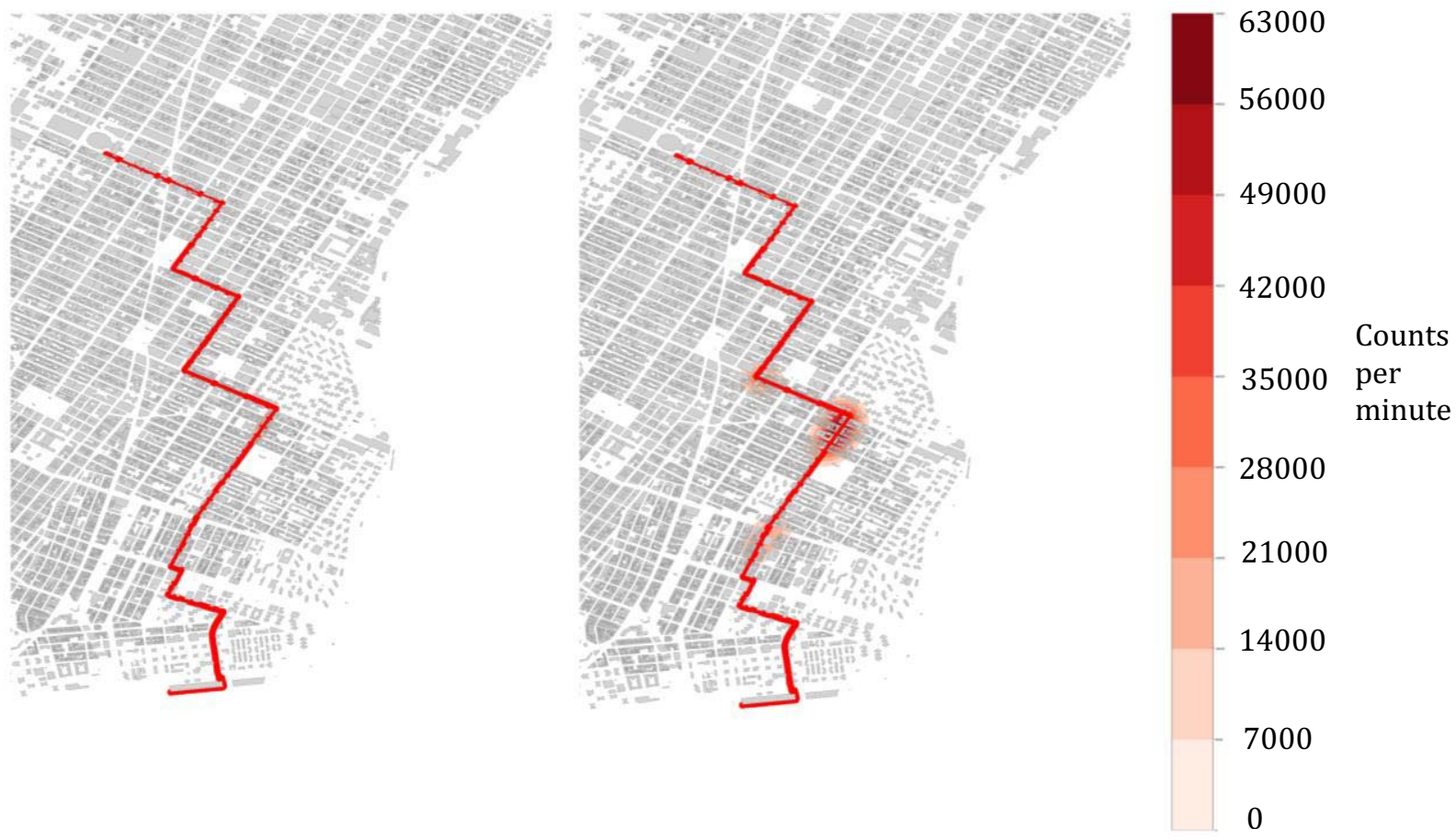

Figure 3. Detection heat maps. Maps show counts per minute detected for a 400 -vehicle mobile detector array of a $0.1 \mathrm{Ci} \mathrm{Co-60} \mathrm{(left)} \mathrm{and} \mathrm{a} 0.5 \mathrm{Ci}$ Co-60 (right) source moving at $2 \mathrm{~m} / \mathrm{s}$. 

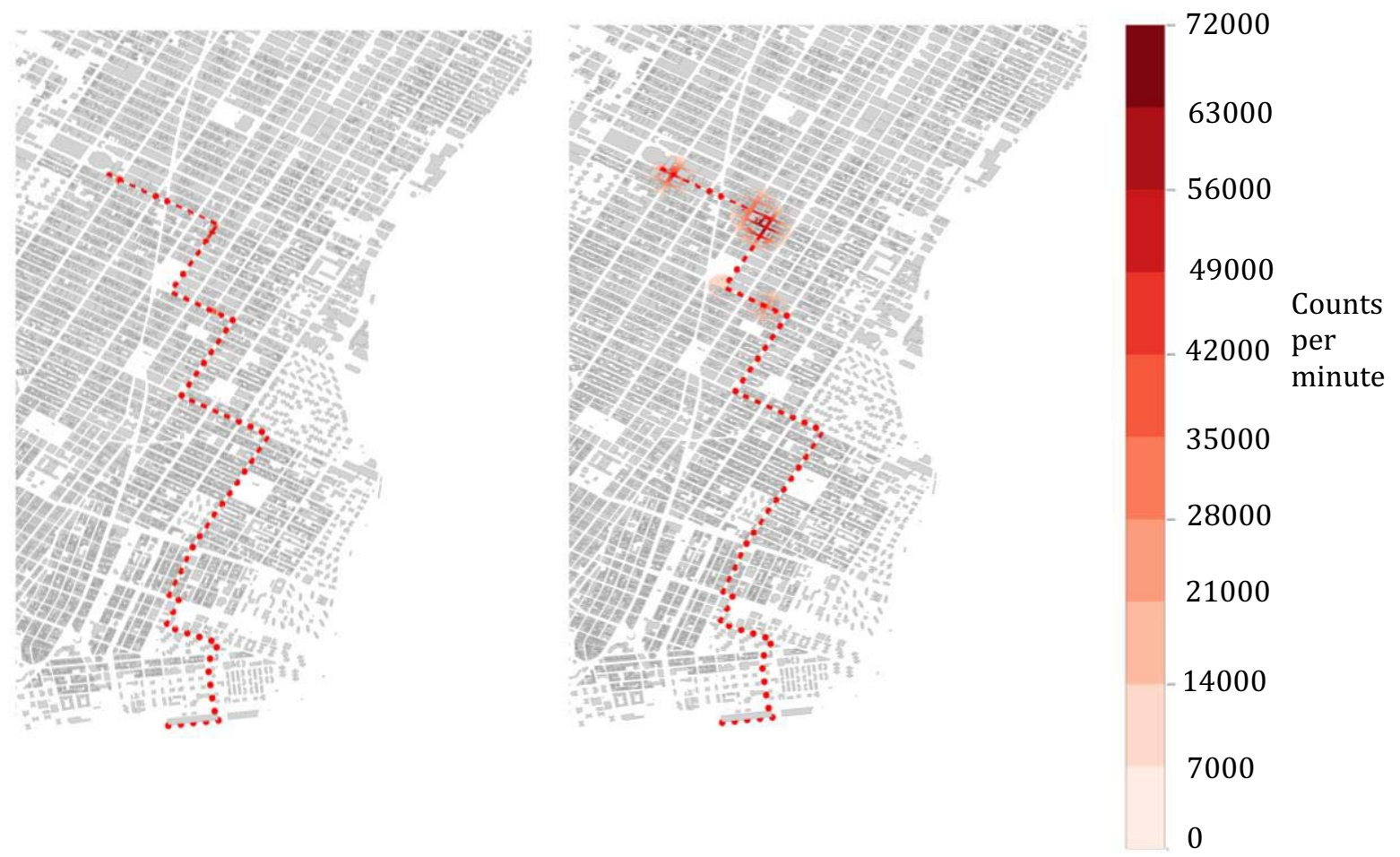

Figure 4. Detection heat maps. Maps show counts per minute detected for a 400 -vehicle mobile detector array of a $0.1 \mathrm{Ci} \mathrm{Co}-60$ (left) and a $0.5 \mathrm{Ci} \mathrm{Co}-60$ (right) source moving at $10 \mathrm{~m} / \mathrm{s}$.

In Cases 1,2,3 the count rate was computed and mapped for a fleet of 200 mobile sensors and a $0.1 \mathrm{Ci}$ source, with source speed from 2 to $15 \mathrm{~m} / \mathrm{s}$. Counts were only recorded when the source moved at $10 \mathrm{~m} / \mathrm{s}$ for a total of $2.35 \times 10^{5}$ counts. The left image in Figure 2 shows the heat map for Case $2(10 \mathrm{~m} / \mathrm{s})$. A small number of counts is detected in the region of the last turn. The low detection in these three cases stems from a weaker source, resulting in a smaller detection radius, as well as low sensor density.

Cases 4,5,6 were evaluated for 200 mobile sensors and a $0.5 \mathrm{Ci}$ source, with source speed from 2 to 15 $\mathrm{m} / \mathrm{s}$. Here we found a similar situation to the cases $1,2,3$. The 2 and $15 \mathrm{~m} / \mathrm{s}$ cases $(4,6)$ showed no detection of the source. However, there is an improvement in the detection between cases 2 and 5 due to a factor of five increase in source strength, from 0.1 to $0.5 \mathrm{Ci}$. The integrated count value in Case 5 is $1.74 \times 10^{6}$, which is more than a factor of five larger than Case $2\left(2.35 \times 10^{5}\right.$ counts $)$. This is because a stronger source increases the minimum detection radius, resulting in more detectors being in range to measure a signal.

In Cases 7,8,9, 400 detectors were simulated with a $0.1 \mathrm{Ci}$ source with source speed varying from 2 to 15 $\mathrm{m} / \mathrm{s}$. With 400 mobile detectors, $8.37 \times 10^{5}$ counts were recorded when the source moved at $2 \mathrm{~m} / \mathrm{s}$, Case 7 . The number of detections in Case 8 is lower than that in Case 2 (left side image Fig 2 vs Fig 4), however the overall integrated count value is greater. This is due to each set of routes (200 versus 400$)$ being unique in the simulations performed. When the source moved at $15 \mathrm{~m} / \mathrm{s}$, Case 9 , zero counts were recorded using 400 detectors, as occurred in Case 2.

Finally, Cases $10,11,12$ were simulated 400 detectors using a $0.5 \mathrm{Ci}$ with speed ranging from 2 to $15 \mathrm{~m} / \mathrm{s}$. $2.31 \times 10^{7}$ and $8.13 \times 10^{6}$ counts were recorded when the source moved at $2 \mathrm{~m} / \mathrm{s}$ (Case 10) and $10 \mathrm{~m} / \mathrm{s}$ (Case 11). The overall count values are greater than a factor of five larger than for Cases 7 and 8 , which are 
equivalent other than the source strength being $0.1 \mathrm{Ci}$. This is for the same reason as the increase in counts between Cases 5 and 2. Case 11 is equivalent to Case 5 other than the number of mobile sensors being increased from 200 to 400 , resulting in more coincidences between source and detectors. The difference between these two cases can be seen in the right-side image of Figures 2 and 4 . In all cases with a source traveling at $15 \mathrm{~m} / \mathrm{s}$, all detectors registered zero counts.

\section{CONCLUSIONS}

This work suggests that mobile sensors on vehicle platforms without explicitly designated routes such as ride share vehicles could be used to locate radioactive sources in a city. While larger sources and detector numbers improve detection, the effect of source speed is not as clear. Increasing source speed would be expected to decrease the overall likelihood of detection as there is less time in which the source is moving. While increasing the speed from $10 \mathrm{~m} / \mathrm{s}$ to $15 \mathrm{~m} / \mathrm{s}$ followed this trend, there was greater detection in the $10 \mathrm{~m} / \mathrm{s}$ cases than the $2 \mathrm{~m} / \mathrm{s}$ cases. Because the results were based on only 200 and 400 sampled routes, this could be the result of variance in the spatial and temporal detector coverage causing fewer coincidences between source and detectors. Repeating the simulations with routes sampled from multiple days' Uber data would be a simple way to estimate the variance.

A systematic study of the sensor network's effectiveness can be done by repeating simulations with varying parameters. The maximum network size of 400 sensors investigated in this work could be increased up to 3500 for the time period simulated. Further work should also investigate the scenarios at different times of day to account for varying levels of traffic, which will impact both vehicle speed and vehicle density. The radiation transport component should be validated using a radiation transport code such as MCNP and incorporating vehicle traffic between the source and detectors would add significantly more realism into the study. While strong gamma emitting 0.5 and $0.1 \mathrm{Ci}^{60} \mathrm{Co}$ sources were used in this work, realistic situations could involve much weaker sources, as well as beta and alpha emitters such as ${ }^{210} \mathrm{Po}$ and ${ }^{90} \mathrm{Sr}$ which would be significantly more difficult to detect. Our method can be extended in a simple way, however, to establish whether detection of these types of emitters is feasible. Incorporating stationary sensors, and detectors of varying types mounted on a range of portable platforms such as Unmanned Aerial Vehicles and hand-held devices could dramatically improve the sensor network's effectiveness, and can be readily implemented into our existing analysis tools.

\section{REFERENCES}

1. Beck, P., et al. ITRAP-International laboratory and field test site exercise for radiation detection instruments and monitoring systems at border crossings. No. IAEA-CN--86. 2001.

2. Shattan, Michael B. An analytic-deliberative process for the selection and deployment of radiation detection systems for shipping ports and border crossings. Diss. Massachusetts Institute of Technology, 2008.

3. Kouzes, Richard T. "Neutron and gamma ray detection for border security applications." $20101 s t$ International Nuclear \& Renewable Energy Conference (INREC). IEEE, 2010.

4. Wein, Lawrence M., and Michael P. Atkinson. "The last line of defense: Designing radiation detection-interdiction systems to protect cities from a nuclear terrorist attack." IEEE Transactions on Nuclear Science 54.3 (2007): 654-669.

5. Liu, Annie H., Julian J. Bunn, and K. Mani Chandy. "Sensor networks for the detection and tracking of radiation and other threats in cities." Proceedings of the 10th ACM/IEEE International Conference on Information Processing in Sensor Networks. IEEE, 2011.

6. King, Michael J., et al. "An urban environment simulation framework for evaluating novel distributed radiation detection architectures." 2010 IEEE International Conference on Technologies for Homeland Security (HST). IEEE, 2010. 
7. Liu, Zheng, and Clair J. Sullivan. "Urban source detection with mobile sensor networks enhanced with machine learning algorithms." 2016 IEEE Nuclear Science Symposium, Medical Imaging Conference and Room-Temperature Semiconductor Detector Workshop (NSS/MIC/RTSD). IEEE, 2016.

8. M. Chandy, C. Pilotto, R. McLean, "Networked sensing systems for detecting people carrying radioactive material", 5th International Conference on Networked Sensing Systems 2008, pp. 148$155,2008$.

9. B. Deb, "Iterative estimation of location and trajectory of radioactive sources with a networked system of detectors", IEEE Transactions on Nuclear Science, vol. 60, no. 2, pp. 1315-1326, 2013.

10. Haklay, Mordechai, and Patrick Weber. "Openstreetmap: User-generated street maps." IEEE Pervasive Computing 7.4 (2008): 12-18.

11. NYS TLC, https://www1.nyc.gov/site/tlc/about/tlc-trip-record-data.page, 7/12/2019

12. Pyroutelib3, https://pypi.org/project/pyroutelib3/

13. Gillies, Sean. "The Shapely user manual." (2013).

14. Rosoff, Heather, and Detlof Von Winterfeldt. "A risk and economic analysis of dirty bomb attacks on the ports of Los Angeles and Long Beach." Risk Analysis: An International Journal 27.3 (2007): 533546.

15. Frost, Robin M. "Dirty bombs: radiological dispersal and emission devices." The Adelphi Papers 45.378 (2005): 75-78.

16. Connell, Leonard W. Dirty Bomb Risk and Impact. No. SAND2017-9121R. Sandia National Lab.(SNL-NM), Albuquerque, NM (United States), 2017.

17. Rees, Brian, et al. RadNet: Open network protocol for radiation data. No. LA-UR-98-3163; CONF980733-. Los Alamos National Lab., NM (United States), 1998. 